\title{
A study of the Joule and Lorentz inputs in the production of atmospheric gravity waves in the upper thermosphere
}

\author{
R. L. Balthazor ${ }^{1}$, R. J. Moffett $^{1}$, G. H. Millward $^{2}$ \\ ${ }^{1}$ Upper Atmosphere Modelling Group, School of Mathematics and Statistics, University of Sheffield, Sheffield S3 7RH, UK \\ ${ }^{2}$ CIRES/University of Colorado, NOAA Space Environment Laboratory, 325 Broadway, Boulder, CO 80303, USA
}

Received: 15 March 1996 / Revised: 12 February 1997 / Accepted: 24 February 1997

\begin{abstract}
First results of a modelling study of atmospheric gravity waves (AGWs) are presented. A fullycoupled global thermosphere-ionosphere-plasmasphere model is used to examine the relative importance of Lorentz forcing and Joule heating in the generation of AGWs. It is found that Joule heating is the dominant component above $110 \mathrm{~km}$. The effects of the direction of the Lorentz forcing component on the subsequent propagation of the $\mathrm{AGW}$ are also addressed. It is found that enhancement of zonal $E \times B$ forcing results in AGWs at F-region altitudes of similar magnitudes travelling from the region of forcing in both poleward and equatorward directions, whilst enhancement of equatorward meridional $E \times B$ forcing results in AGWs travelling both poleward and equatorward, but with the magnitude of the poleward wave severely attenuated compared with the equatorward wave.
\end{abstract}

\section{Introduction}

The production and propagation mechanisms of atmospheric gravity waves (AGWs) and associated travelling ionospheric disturbances (TIDs) have been studied in great detail; reviews have been written by e.g. Hines (1960), Hines (1974), Francis (1975), Richmond (1978), Hunsucker (1982) and most recently by Jing and Hunsucker (1993). In summary, three mechanisms which lead to the formation of an AGW in the auroral zone are (1) Joule heating (frictional heating of the neutral gas due to a difference in velocity between the neutrals and the ions), (2) Lorentz momentum forcing (momentum transfer between the neutral gas and the ions due to collisions, synonymous with ion drag), and (3) heating due to particle precipitation. However, the

Correspondence to: R. L. Balthazor relative importance of each mechanism is less certain (Williams et al., 1988).

Chimonas and Hines (1970) presented an analytical calculation of the generation of AGWs and TIDs in an ideal inviscid isothermal windless atmosphere, using a two-dimensional auroral electrojet source function that produced a pressure perturbation in the atmosphere. An assumption was made that the wave frequency $\omega$ is less than the Brunt-Väisälä frequency (thus ignoring acoustic wave solutions). Chimonas and Hines (1970) gave an expression for the relative importance of the Joule and Lorentz terms in the production of the AGW as

$\frac{\text { Lorentz contribution }}{\text { Joule contribution }}=\frac{L}{J}=\frac{g H B_{z} \sigma_{c}(z)}{C_{L} j(z)}$

where $g$ is the gravitational acceleration, $H$ is the atmospheric scale height, $B_{z}$ is the vertical component of the magnetic field, $C_{L}$ is a characteristic wave speed, $\sigma_{c}$ is the Cowling conductivity and $j$ is the current density. Note that in the auroral region, the magnetic field lines are effectively vertical so $B_{z} \approx B$. Using values of $g=9.5 \mathrm{~m} \mathrm{~s}^{-2}, H=10^{4} \mathrm{~m}, B_{z}=5.4 \cdot 10^{-5} \mathrm{~T}$ and $C_{L}=$ $350 \mathrm{~ms}^{-1}$, they obtained:

$\frac{L}{J} \simeq 15 \cdot \frac{\sigma_{c}}{j}$

(where $j$ is in $\mathrm{mA} \mathrm{m}^{-1}$ ). Chimonas and Hines (1970) state that for an electric field of $\approx 60 \mathrm{mV} / \mathrm{m}$, uncertainties in the value of $j$ lead to a variation in the value of $L / J$ of 0.1 to 10.0 .

Brekke (1979) used height-dependent values of $j$ and $\sigma_{c}$, re-applying them to the equation of Chimonas and Hines (1970), to yield

$\frac{L}{J} \simeq \frac{g H B_{z}}{C_{L}} \sqrt{\frac{\omega^{2}+v^{2}(z)}{\omega^{2}}} \frac{1}{E}$

where $\omega$ is the ion gyrofrequency and $v(z)$ is the ionneutral collision frequency. Inserting the values of $g, H, B_{z}$ and $C_{L}$ used by Chimonas and Hines (1970), we obtain 
$\frac{L}{J} \simeq 15 \cdot \sqrt{\frac{\omega^{2}+v^{2}(z)}{\omega^{2}}} \frac{1}{E}$

where $E$ is given in $\mathrm{mV} / \mathrm{m}$. This yields smaller values of $L / J$ than those obtained by Hunsucker (1977) due to the decreased Cowling conductivity above $120 \mathrm{~km}$. Brekke (1979) states that 'For reasonable electric field strength say above $40 \mathrm{mV} / \mathrm{m}$ the ratio $L / J$ will never exceed 1 above $120 \mathrm{~km}$ '. It is noted however that Brekke's (1979) analysis is confined to the E-region.

Richmond (1978) used a combination of analytical and numerical techniques, obtaining wave-like solutions of the equations of energy, momentum and continuity in a non-isothermal atmosphere. Although the approach is similar to that of Chimonas and Hines (1970) the source perturbation used was an enhancement in the electron density at E-region altitudes, yielding an expression for $L / J$ of

$\frac{L}{J}=\left[\frac{\bar{N}(107-121 \mathrm{~km})}{\bar{N}(121-148 \mathrm{~km})} \frac{187 \mathrm{~ms}^{-1}}{E_{x} / B}\right]^{2}$

where $\bar{N}$ is the mean electron density over the specified height range. Joule heating is dominant in the upper altitude region producing waves with mean velocity $\sim 640 \mathrm{~ms}^{-1}$, whereas Lorentz forcing is dominant in the lower altitude regime producing waves with a lower velocity of $\sim 320 \mathrm{~ms}^{-1}$ (Hunsucker, 1982).

Data from the Chatanika incoherent scatter radar during an impulsive E-region electric field event was applied by Hunsucker (1977) to Eq. (1). Magnetometer data was used to obtain the height-integrated current density $j$ whilst Hall and Pedersen conductivity observations (Brekke et al., 1974) were used to calculate more precise values for the Cowling conductivity. A mean value for $L / J$ of 2.37 was obtained for an electric field of $\approx 43 \mathrm{mV} / \mathrm{m}$ (Hunsucker, 1977); using the equations of Richmond and data from the same event, a value of $L / J$ of 3.13 was obtained (Hunsucker, 1982).

The model of Chimonas and Hines (1970) (upon which the calculations of Brekke, 1979 and Hunsucker, 1977 are based), assumes an atmosphere perturbed only in pressure. Jing and Hunsucker (1993) presented an analytical solution to take into account density, pressure and velocity perturbations, although the simplifications of an ideal windless isothermal atmosphere ignoring Coriolis effects are retained. The general solution shows that Joule heating terms are more effective at generating large scale $\mathrm{AGW}$ s that propagate to lower latitudes, whereas Lorentz terms are more effective at generating medium scale AGWs.

It must be noted that the previous studies have assumed the altitude of the effective source region is in the E-region and that AGWs generated propagate outwards and upwards, reflecting off lower layers of the thermosphere. (Thus, the disturbance seen at a distant point is an interference summation of both directly propagating waves and reflected waves.) No calculations have directly addressed source mechanisms at higher altitudes where several important differences become apparent. Above about $130 \mathrm{~km}$, the Hall con- ductivity becomes small compared to the Pedersen conductivity, so $\sigma_{C} \approx \sigma_{P}$ and $J=\sigma_{P} E$. Furthermore, the direction of the ion drift due to the electric field is dependent on the ratio $k_{i}$ of ion gyrofrequency to collision frequency; in the E-region, $k_{i} \ll 1$ and the iondrift $V_{i}$ tends to be aligned with the applied E-field; in the F-region, $k_{i} \gg 1$, and the ion drift $V_{i}$ tends to be perpendicular to both $E$ and $B ; V_{i}^{\perp} \approx E \times B / B^{2}$.

\section{Model description}

The global model of the coupled thermosphere/ionosphere/plasmasphere has been described by Millward et al., (1993b) and more extensively by Fuller-Rowell et al., (1996) and Millward et al., (1996). In brief, coupled equations of momentum, energy and continuity are solved at fixed grid points to calculate values of density, temperature, and velocity of the neutral atmosphere, and of the $\mathrm{O}^{+}$and $\mathrm{H}^{+}$ions in open flux tubes at high latitudes, and closed flux tubes in the plasmasphere. The closed flux tubes are aligned along an eccentric dipole approximation to the Earth's magnetic field, arranged such that each flux tube returns to its starting position in a 24 hour period. Concentrations of $\mathrm{N}^{+}, \mathrm{O}_{2}^{+}, \mathrm{NO}^{+}$and $\mathrm{N}_{2}^{+}$are derived from chemical equilibrium considerations. The model resolution is $2^{\circ}$ in latitude and $18^{\circ}$ in longitude, co-rotating with the Earth and defining a spherical polar coordinate system. Vertically, the thermosphere is output at fifteen fixed pressure levels, each at a separation of one scale height and the lowest at a boundary defined to be $1 \mathrm{~Pa}$ at 80 $\mathrm{km}$. The fully-coupled thermosphere-plasmasphere-ionosphere model takes into account non-uniform temperature and wind, the viscous nature of the atmosphere, and Coriolis effects. Thus we may use it to more directly address the source mechanisms of AGWs at F-region altitudes.

The model was run until steady-state equilibrium (in a diurnal sense) was obtained, for January 1 conditions. A Foster electric field model (Foster, 1983) was used with an $\mathrm{F}_{10.7}$ index of 165 and TIROS precipitation activity level $7\left(\mathrm{~K}_{P} \sim 3\right)$ (Fuller-Rowell and Evans, 1987), and these were fixed during the simulations. This steady-state atmosphere was used as input conditions for the study, which examined the effects on the atmosphere during the period 12 UT to 17 UT (the start time constrained by the form of empirical electric field data used, and the end time chosen to allow study of the gravity waves propagating to equatorial latitudes).

A disturbance in the atmosphere was created by introducing a geographically co-rotating enhanced electric field over a localised area in the near-midnight sector of both northern and southern auroral zones, to simulate a simultaneous enhancement of the auroral electrojet. The extent of the enhanced electric field was $72^{\circ}$ zonally $(\sim 2500 \mathrm{~km})$ and $10^{\circ}$ meridionally $(\sim 1100$ $\mathrm{km}$ ) with a ramped distribution in both latitude and longitude; Table 1 shows the magnitude of the enhancement to the electric field in the Northern Hemisphere, 
Table 1. Electric field enhancements (in $\mathrm{mV} / \mathrm{m}$ ) at specified grid points in the Northern Hemisphere

\begin{tabular}{lclclc}
\hline & $126^{\circ} \mathrm{E}$ & $144^{\circ} \mathrm{E}$ & $162^{\circ} \mathrm{E}$ & $180^{\circ} \mathrm{E}$ & $198^{\circ} \mathrm{E}$ \\
\hline $74^{\circ} \mathrm{N}$ & 6 & 12 & 20 & 12 & 6 \\
$72^{\circ} \mathrm{N}$ & 21 & 42 & 70 & 42 & 21 \\
$70^{\circ} \mathrm{N}$ & 30 & 60 & 100 & 60 & 30 \\
$68^{\circ} \mathrm{N}$ & 30 & 60 & 100 & 60 & 30 \\
$66^{\circ} \mathrm{N}$ & 21 & 42 & 70 & 42 & 21 \\
$64^{\circ} \mathrm{N}$ & 6 & 12 & 20 & 12 & 6 \\
\hline
\end{tabular}

with a similar distribution in the Southern Hemisphere. Both enhancements were centred on the geographic line of longitude $162^{\circ} \mathrm{E}$ to lie in the midnight sector (where there would be a meridional wind originating from the two-cell polar convection pattern); to coincide with the auroral zones, the northern enhancement was centred at $69^{\circ} \mathrm{N}$ geographic and the southern at $53^{\circ} \mathrm{S}$ geographic. The instantaneous $E \times B$ ion drift velocity corresponding to an electric field enhancement of $100 \mathrm{mV} / \mathrm{m}$ is about $2 \mathrm{~km} \mathrm{~s}^{-1}$ in the F-region. The duration of the electric field enhancement was 10 minutes from 1215 UT. The magnitude and duration of the enhanced electric field was designed to simulate a typical 'burst' in the auroral ion velocity (e.g. Millward et al., 1993a), whilst the extent was designed to cover a significant fraction of the auroral region and to cover several 'grid points' on the thermospheric grid to avoid resolution effects; the choice of 6 by 5 grid points was arbitrary.

The orientation of the enhanced electric field was adjustable and two separate computations were carried out: (1) with the enhanced electric field meridional and equatorwards (thus producing F-region ions drifts zonally and eastwards); and (2) with the enhanced electric field zonal and westwards (thus producing Fregion ion drifts meridional and equatorwards). A third simulation of January 1 conditions with no enhanced electric field was used as a control comparison. The Joule heating and Lorentz forcing components were calculated by

Joule heating $=J=J \cdot E^{\prime} \equiv J \cdot\left(E+\left(V_{n} \times B\right)\right)$

Lorentz forcing $=L=V_{n} \cdot(J \times B)$

It is noted that in this formulation, the total heating $J+L$ is given by

$J+L=J \cdot E$

\section{Results}

The AGW is essentially a pressure wave travelling through the thermosphere and thus may be characterised directly by its effect on a fixed pressure level in the atmosphere. Observed characteristics more commonly used in AGW studies such as temperatures and winds are secondary effects of the pressure wave travelling through the thermosphere, whereas the change in height of a fixed pressure level is a more 'direct' measure of the wave. The effect on the thermosphere has been charac- terised here by examining the change in the height of a fixed pressure level labelled H12. This lies 11 scale heights above $1 \mathrm{~Pa}$, corresponding to an altitude varying globally between 260 and $290 \mathrm{~km}$ (below the F2 peak). To compare with more commonly observed quantities, Fig. 1 shows both the change in height of a fixed pressure level and the change in neutral temperature (from diurnal steady state) at 12.20 UT during a zonal electric field enhancement and at $12.50 \mathrm{UT}$, when the AGW is propagating southwards. The maximum temperature change during the electric field enhancement is of the order of $60 \mathrm{~K}$ (a fractional change of about 0.07 ).

Figure 2 shows the effect of the disturbance on the height of the fixed pressure level H12 for the case of a meridional enhanced electric field (Fig. 2a), and a zonal enhanced electric field (Fig. 2b). In the first case, the F-region ion-drift is zonal and eastwards; in the second, meridional and equatorwards. At F-region altitudes, the wave is formed within 5 minutes of the onset of the enhanced electric field. Details of in situ effects caused by an enhanced electric field (and the effects of the consequent TIDs), using the coupled model, have been discussed by Millward et al. (1993a, b). In the region of enhanced electric field (the source region) it was found that ion-neutral frictional heating caused an expansion of the atmosphere, loss of plasma due to increased recombination rate and upward flow, and increased abundance of molecular ions. The initial expansion of the atmosphere was followed by a relaxation, leading to the formation of a large-scale AGW (Millward et al., 1993a). Here we see that both cases result in AGWs propagating principally meridionally from the source

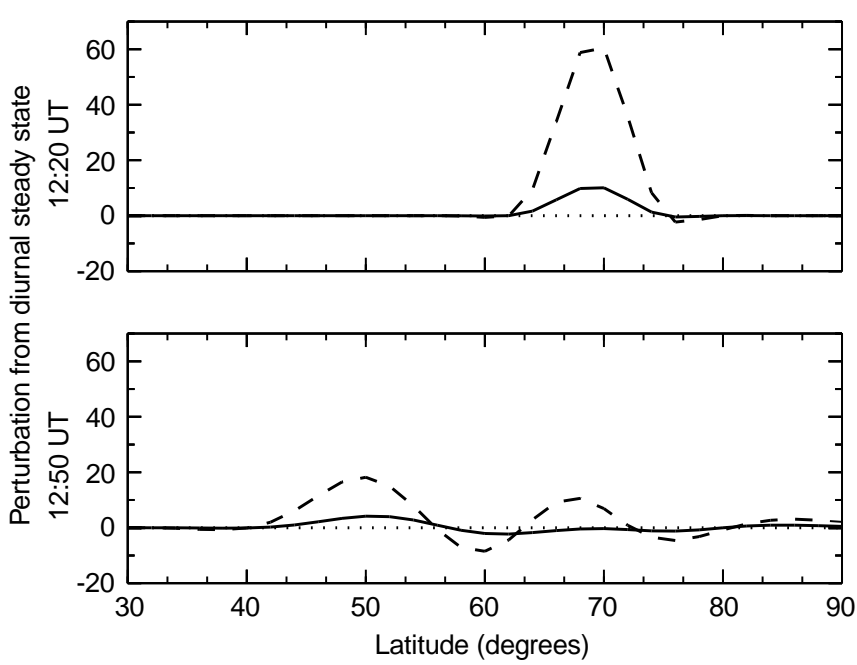

Fig. 1. Perturbations in the height of a fixed pressure level and in neutral temperature from diurnally steady-state conditions. The upper panel gives results for 12.20 UT during a zonal electric field enhancement, and the lower panel for 12.50 UT as the resultant AGW propagates southwards. The solid line shows the change in height of a fixed pressure level at approximately $260 \mathrm{~km}$ (with the vertical scale reading as $\mathrm{km}$ ), while the dashed line shows the change in neutral temperature along that fixed pressure level (with the vertical scale reading as degrees Kelvin). The horizontal dotted line shows the undisturbed steady-state conditions for both quantities (i.e., no perturbation) 


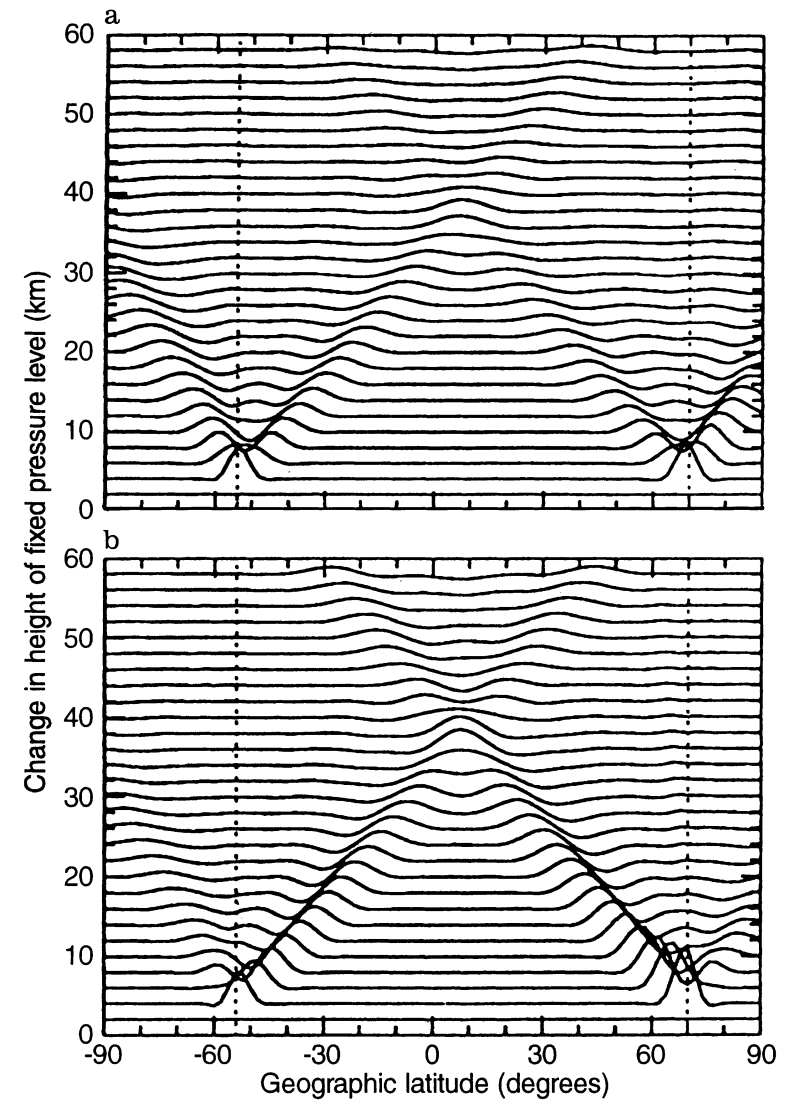

Fig. 2a,b. The change in the height of the fixed pressure level H12 plotted against latitude on the line of longitude $162^{\circ} \mathrm{E}$, due to a short duration localised enhanced electric field a meridionally, and $\mathbf{b}$ zonally. The change in the height of the fixed pressure level has been shown at 30 consecutive intervals, each $10 \mathrm{~min}$ apart from $t=0$ to $t=290 \mathrm{~min}$, each successive plot beyond the first displaced $2 \mathrm{~km}$ on the vertical axis for clarity. The enhanced electric field disturbance lasted from $t=15$ to $t=25 \mathrm{~min}$, and the locations of the centres of enhancement of the electric field are shown by vertical broken lines

region. The equatorward propagating waves propagate with approximately constant velocity and interfere when they meet, each passing through into the other hemisphere. In the first case, the simulated equatorwardtravelling waves have velocities of $680 \mathrm{~ms}^{-1}$ and $670 \mathrm{~ms}^{-1}$ for Northern and Southern-Hemisphere originating waves respectively; in the second case, $700 \mathrm{~ms}^{-1}$ and $690 \mathrm{~ms}^{-1}$ respectively. The waves are identified in each case as large-scale imperfectly ducted gravity wave modes (Francis, 1973). As this work deals with the Joule and Lorentz heating effects in the source region, the propagation and dispersion characteristics of the modelled AGWs will not be discussed here.

There is negligible zonal propagation of the AGWs in the simulation results presented here. It is noted that the resolution of the model in the zonal direction $\left(18^{\circ}\right)$ is relatively coarse; it is uncertain how much effect this has on the direction of propagation. Furthermore, there are modelled physical effects that are believed to contribute to the anisotropy of propagation. First, for the results presented here, the source region is considerably extended in the zonal direction compared to the meridional direction (Table 1). The influence of the geometry of the source region has been tested by carrying out model runs with a point source and with a meridionally extended source (results not shown here). It was found that some zonal propagation occurred but that propagation in the meridional direction was dominant. Secondly, the effect of ion-drag in the F-region is different in the meridional and zonal directions. In the former direction, the ions can respond to neutral air motion by diffusing along the field lines; in the latter, the ions are bound to the field lines.

Figure 3 shows the ratio of $L / J$ calculated at 13 fixed pressure levels $\mathrm{H}=5$ (corresponding to four scale heights above $80 \mathrm{~km}$, about $104 \mathrm{~km}$ ), to $\mathrm{H}=14$, (about $380 \mathrm{~km}$ ). The solid lines show the ratio $L / J$ (calculated form Eqs. 6 and 7) at latitude $70^{\circ} \mathrm{N}$, longitude $162^{\circ} \mathrm{E}$, $2330 \mathrm{LT}$, for (a) a quiet atmosphere, and (b) an atmosphere with an enhanced meridional $100 \mathrm{mV} / \mathrm{m}$ electric field. Our modelled results show that for a quiet atmosphere $L / J$ is approximately 0.8 above about 110 $\mathrm{km}$, and the Lorentz force becomes dominant below that altitude. Increasing the local electric field to $100 \mathrm{mV} / \mathrm{m}$ in a southward direction decreases $L / J$ at all heights by approximately an order of magnitude.

The results obtained by calculation by Brekke (1979) (Eq. 3) are displayed in Fig. 3 as three discrete points for a $100 \mathrm{mV} / \mathrm{m}$ electric field in a stationary atmosphere. Whilst Brekke's (1979) three points show an increase of $L / J$ below $120 \mathrm{~km}$, they are larger than results obtained from the coupled model by up to an order of magnitude. It is strongly emphasised that the ratio $L / J$ as obtained in this study is derived from the coupled terms in the energy equation. It does not require a priori knowledge of the wave speed, and the quantities are fully dynamic in height and time. Brekke's (1979) analysis was based on a stationary neutral atmosphere with assumptions of a fixed gyrofrequency of $172 \mathrm{~Hz}$, wave speed of

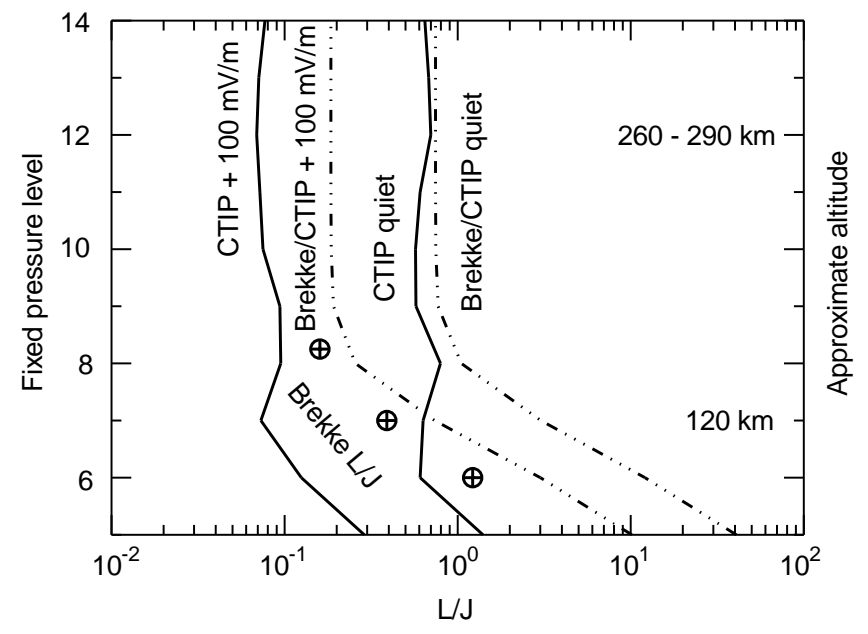

Fig. 3. Plot of the ratio of Lorentz forcing to Joule heating against height at latitude $70^{\circ} \mathrm{N}$, longitude $162^{\circ} \mathrm{E}$, Day 1 . The two solid lines show the coupled model results for (a) quiet atmosphere, and (b) during the $100 \mathrm{mV} / \mathrm{m}$ enhanced electric field. The broken lines show results from the extrapolation of the Brekke formula, Eq. (4), for both quiet and enhanced atmospheres, whilst the three discrete points are the Brekke results 
$350 \mathrm{~ms}^{-1}$ and scale height of $10 \mathrm{~km}$. Moreover, Brekke's (1979) stationary neutral atmosphere is likely to underestimate the Joule heating term (Eq. 7) and thus overestimate $L / J$ in this case, where the background neutral wind is principally meridional and F-region ion drifts in the source region (due to the enhanced electric field) are zonal.

Also shown in Fig. 3 as broken lines are the results of applying model-derived dynamic values of $\omega, v_{n i}$ and $E$ to Eq. (4) (Brekke, 1979), extrapolating the formula to higher altitudes. At low altitudes, Brekke's (1979) formula (Eq. 3) is likely to further overestimate $L / J$ due to the a priori assumption of a lower wave speed than obtained in the fully coupled model. Therefore, we expect the result that Brekke's (1979) calculations will yield a large $L / J$ particularly at lower altitudes.

The dominance of Joule heating in the F-region for an enhanced electric field is also compared to the deduction by Williams et al., (1988); from EISCAT incoherent scatter radar observations taken during a period of moderate electric field disturbances (up to about $60 \mathrm{mV} / \mathrm{m}$ in a dominantly southward direction). Williams et al. (1988) stated that 'The Lorentz force is again dominant below $110 \mathrm{~km}$, but the ratio $L / J$ is closer to unity at greater heights'. It must be noted however that Williams et al. (1988) calculated $L / J$ using Brekke's (1979) formula, and inserting observed values of the parameters. The electric field and wave velocity used were both smaller than that measured in our simulations, both effects tending to increase the value of $L / J$ calculated by Williams et al. (1988) and we suggest that this is the main source of the difference between the statement of Williams et al. (1988) and our results.

It must be noted that the effects of Joule heating and Lorentz forcing cannot be fully separated in the model; both heating mechanisms are coupled to the neutral wind vector $V_{n}$ which will be altered by heating of the thermosphere. However, we may partially separate the effects of the two mechanisms. We denote the Joule heating and Lorentz forcing in the absence of an enhanced electric field by $J_{0}$ and $L_{0}$, and in the presence of an enhanced electric field by $J_{E}$ and $L_{E}$. If, when the enhanced electric field is applied, one of the mechanisms is artificially held at equilibrium values $J_{0}$ or $L_{0}$, we obtain either

$L^{\prime}=L_{E} \quad$ and $\quad J^{\prime}=J_{0}+J_{L}$

or

$J^{\prime}=J_{E} \quad$ and $\quad L^{\prime}=L_{0}+L_{J}$

where $J_{L}$ is Joule heating resulting from the change in the neutral wind due to the additional Lorentz forcing, and $L_{J}$ is Lorentz forcing resulting from a change in the neutral wind due to the additional Joule heating:

$J_{L}=J \cdot\left(\delta V_{n} \times B\right)$

$L_{J}=\delta V_{n} \cdot(J \times B)$

where heating has altered the neutral wind by an amount $\delta V_{n}$. The simulation yielded $J_{L} / J_{E} \approx 0.1$ and $L_{J} / L_{E} \approx 0.25$.
Figures 4 and 5 demonstrate the formation of the AGW at the fixed pressure level H12. Figure 4 shows results with a meridional enhanced electric field: (a) $J^{\prime}=J_{E}, L^{\prime}=L_{E}, \quad$ (b) $J^{\prime}=J_{E}, L^{\prime}=L_{0}+L_{J}$, and (c) $J^{\prime}=J_{0}+J_{L}, L^{\prime}=L_{E}$. Figure 5 shows the effects of the same inputs with a zonal enhanced electric field. In each figure, (a) illustrates the AGW formed by coupled Joule and Lorentz mechanisms, identical to Fig. 2 and shown here for reference.

Figures $4 \mathrm{~b}$ and $5 \mathrm{~b}$ show the AGW produced by reducing $L$ as described. It can be seen that there is little

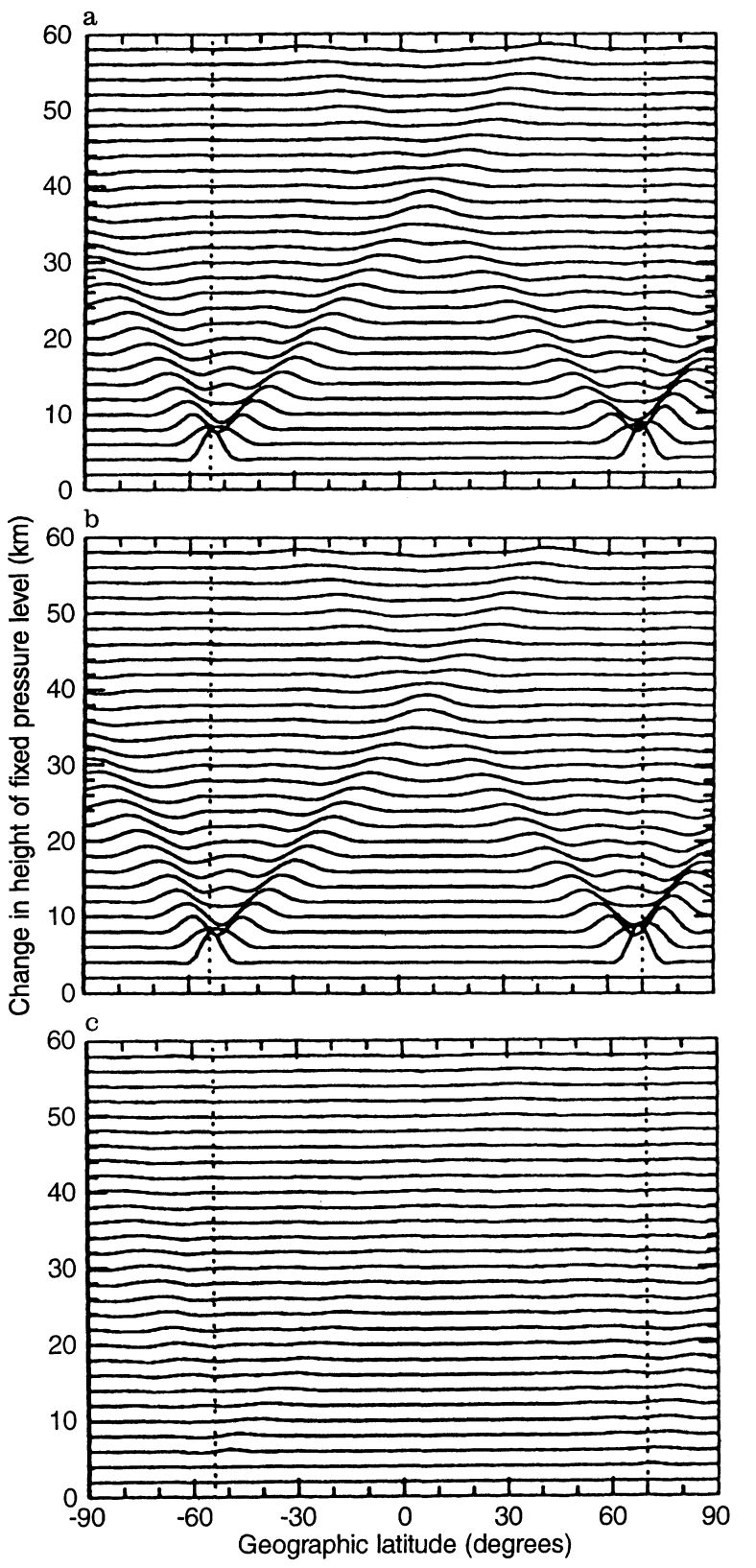

Fig. 4a-c. The change in the height of the fixed pressure level H12 plotted against latitude due to a short duration localised meridional enhanced electric field, with a Joule heating and Lorentz forcing, b Lorentz forcing due to the enhanced electric field artificially removed, c Joule heating due to the enhanced electric field artificially removed. The plotting convention is identical to that of Fig. 2 

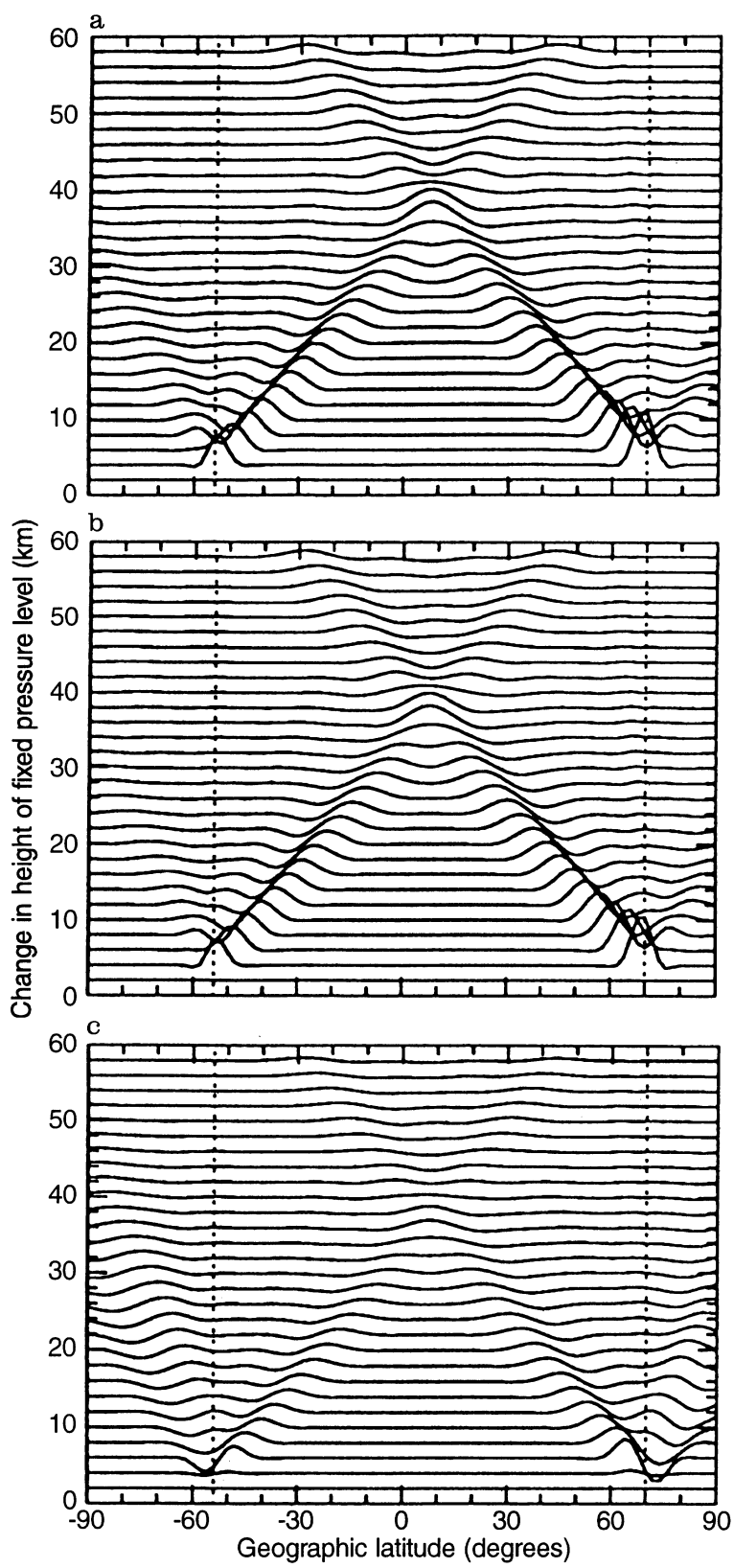

Fig. 5a-c. As for Fig. 4, except that the enhanced electric field is zonal. The plotting convention is identical to that of Fig. 2

change to the resultant $\mathrm{AGW}$, a strong confirmation that Joule heating is the dominant production mechanism in each case.

The effect on the AGW of reducing the Joule heating component $J$ is shown in each case in Figs. 4c and 5c. The Joule heating due to the Lorentz forcing $J_{L}$ is small, and where the enhanced field is meridional (Fig. 4) and thus the F-region ion drift is zonal, there is little AGW production. Conversely, where the enhanced field is zonal (Fig. 5) and the ion drift meridional, an AGW is created propagating mainly equatorially. It is noted that in this case, the main AGW production occurs not at $t=20 \mathrm{~min}$ (whilst the enhanced electric field is applied) but at $t=30 \mathrm{~min}$, after it has reverted to 'normal' values. It is speculated that the high velocity ion drift forces the neutral atmosphere into motion through the Lorentz forcing mechanism; Joule heating is small, and there is little AGW production. When the enhanced electric field is relaxed, the ions revert to former drift patterns, but the neutral atmosphere is still moving south; the resultant Joule heating forms an AGW a little to the south of the centre of the applied electric field. It is noted that the time delay is not due to waves launched at lower altitudes propagating upwards, as the wave formation is delayed at all altitudes from $80 \mathrm{~km}$ upwards (to the top of the model), although for conciseness only the wave formation at around $260 \mathrm{~km}$ is shown. It is also emphasised that this attempt to separate the effects of Joule heating and Lorentz forcing cannot be completely quantitative.

\section{Conclusions}

These first results from the coupled thermosphereionosphere-plasmasphere model suggest that an enhancement in the auroral electric field (either meridional or zonal) produces significant AGW propagation at F-region altitudes. A direct measure of the ratio $L / J$ of the Joule heating and Lorentz forcing mechanisms show that Joule heating is dominant; however, the direction of the ion drift velocity influences the direction of propagation of the AGW. In both cases studied, the dominant Joule heating mechanism produces a wave with velocity about $680 \mathrm{~ms}^{-1}$ propagating equatorwards. Previous analytic and observational predictions of $L / J$ have used several approximations to the atmospheric physics and have observed different AGW formations (under different starting conditions). A direct comparison is therefore difficult to interpret, but these fully dynamic and coupled results of $L / J$ follow, although are smaller by up to an order of magnitude, those of Brekke (1979) and Williams et al. (1988). The discrepancy may be explained by the use of a priori assumptions of atmospheric and AGW parameters in the previous work and more crude approximations than those used in the fully coupled model. $L / J$ is shown to be decreasing with increasing applied electric field. $L / J$ is also approximately constant with height above around $120 \mathrm{~km}$, increasing below that altitude.

Acknowledgements. This work has been supported by the UK Science and Engineering Research Council and Particle Physics and Astronomy Research Council. We thank the referee for constructive comments.

Topical Editor D. Alcaydé thanks a referee for his help in evaluating this paper.

\section{References}

Brekke, A., Relative importance of Joule heating and the Lorentz force in the auroral electrojet, J. Atmos. Terr. Phys., 41, 475, 1979

Brekke, A., J. R. Doupnik, and P. M. Banks, Incoherent scatter measurements of $\mathrm{E}$ region conductivities and currents in the auroral zone, J. Geophys. Res., 79, 3773, 1974.

Chimonas, G., and C. O. Hines, Atmospheric gravity waves launched by auroral currents, Planet. Space Sci., 18, 565, 1970. 
Foster, J. C., An empirical electric field model derived from Chatanika radar data, J. Geophys. Res., 88, 981, 1983.

Francis, S. H., Acoustic-gravity modes and large-scale travelling ionospheric disturbances of a realistic, dissipative atmosphere, J. Geophys. Res., 78, 2278, 1973.

Francis, S. H., Global propagation of atmospheric gravity waves, J. Atmos. Terr. Phys., 37, 1011, 1975.

Fuller-Rowell, T. J., and D. S. Evans, Height-integrated Pederson and Hall conductivity patterns inferred from the TIROSNOAA satellite data, J. Geophys. Res., 92, 7606, 1987.

Fuller-Rowell, T. J., D. Rees, S. Quegan, R. J. Moffett, M. V. Codrescu, and G. H. Millward, A coupled thermosphereionosphere model (CTIM). STEP Handbook on Ionospheric Models, Ed. RW Schunk: Utah State University, 217, 1996.

Hines, C. O., Internal atmospheric gravity waves at ionospheric heights, Can. J. Phys., 38, 1441, 1960.

Hines, C. O., The upper atmosphere in motion, Geophysical Monograph, American Geophysical Union, 1974.

Hunsucker, R. D., Estimate of the relative importance of Joule heating and the Lorentz force in generating atmospheric gravity waves from the auroral electrojet, J. Geophys. Res., 82, 4826, 1977.

Hunsucker, R. D., Atmospheric gravity waves generated in the high-latitude ionosphere: a review, Rev. Geophys. Space Phys., 20, 293, 1982.
Jing, N., and R. D. Hunsucker, A theoretical investigation of sources of large and medium scale atmospheric gravity waves in the auroral oval, J. Atmos. Terr. Phys., 55, 1667, 1993.

Millward, G. H., R. J. Moffett, S. Quegan, and T. J. Fuller-Rowell, Effects of an atmospheric gravity wave on the midlatitude ionospheric F layer, J. Geophys. Res., 98, 19173, 1993a.

Millward, G. H., S. Quegan, R. J. Moffett, T. J. Fuller-Rowell, and D. Rees, A modelling study of the coupled ionospheric and thermospheric response to an enhanced high-latitude electric field event, Planet. Space Sci., 41, 45, 1993b.

Millward, G. H., R. J. Moffett, S. Quegan, and T. J. Fuller-Rowell, A coupled thermosphere-ionosphere-plasmasphere model CTIP, STEP Handbook on Ionospheric Models, Ed. RW Schunk: Utah State University, 239, 1996.

Richmond, A. D., Gravity wave generation, propagation, and dissipation in the thermosphere. J. Geophys. Res., 83, 4131, 1978.

Williams, P. J. S., G. Crowley, K. Schlegel, T. S. Virdi, I. McCrea, G. Watkins, N. Wade, J. K. Hargreaves, T. Lachlan-Cope, H. Muller, J. E. Baldwin, P. Warner, A. P. van Eyken, M. A. Hapgood, and A. S. Rodger, The generation and propagation of atmospheric gravity waves observed during the Worldwide Atmospheric Gravity-wave Study (WAGS), J. Atmos. Terr. Phys., 50, 323, 1988. 